Spinal PA movements behave 'as if' there are limitations of local segmental mobility and are large enough to be perceivable by manual palpation: A synthesis of the literature

Neil Tuttle, BSc, MPhil, PhD

Griffith University

Charles Hazle, BSc, MSc PhD

University of Kentucky 


\begin{abstract}
Background: Posterio-anterior (PA) movements are one type of passive intervertebral movement used to assess and treat perceived deficits in localized segmental mobility. Objectives: To describe: 1) The specific effects that reductions in segmental mobility would be expected to have on PA movements; 2 ) How differences in PA movements in clinical situations compare to what would be expected with reduced segmental mobility; and 3) Whether such differences in PA movements are likely to be perceivable by manual palpation. Methods: Multiple modelling studies and in vivo measurements of PA movements are described. Results: The findings indicate the differences in PA movements present in clinical conditions corresponds with the differences that would be expected with decreased segmental mobility. The differences both predicted from the modelling and found in clinical conditions were greatest at low levels of force. Additionally, the differences are large enough that individuals with training are likely to be capable of 1) consistently producing controlled movements with sufficiently small magnitudes of force to assess the movements, and 2) detecting the differences in stiffness expected from modelling and found in clinical situations. Conclusions: Implications for clinical practice and teaching include the need to attend to the stiffness of PA movements at lower levels of force than those typically described. The authors recommend a three tiered approach to assessment of PA movements which may assist in both clinical practice and teaching manual therapy skills.
\end{abstract}




\section{Spinal PA movements behave 'as if' there are limitations of local segmental mobility and are large enough to be perceivable by manual palpation: a synthesis of research}

\section{Introduction}

Posterio-anterior (PA) movements are one of a range of passive intervertebral movements (PIVMs) used to assess or treat perceived deficits in localised segmental mobility thought to be associated with mechanical pain syndromes. PA movements were originally thought to produce localised accessory movements [1] and are still referred to as Passive Accessory Inter-vertebral Movements. It is now clear however from both in vivo [2-7] and in vitro studies [8-10] that the spinal movement produced by PA movements is neither localised, nor primarily accessory. Rather the movement produced spans a number of intervertebral levels, is primarily physiological movement in the sagittal plane, and is potentially influenced by a number of non-articular factors [3, 5, 11-14]. We suggest empirical evidence exists that PA movements behave 'as if' there is a localised limitation of segmental mobility and the relevant differences are sufficiently large to be perceivable by manual palpation. It is not the intention of this paper to suggest whether restrictions of local segmental mobility exist, what the mechanisms of reduced segmental mobility might be or whether such changes in mobility would even be relevant. Rather this paper will consider the behaviour of PA movements from an empirical perspective and address three questions: 1) If localised deficits of spinal mobility were to occur, what impact would be expected on PA movements; 2) How differences in PA movements in clinical situations compare to what would be expected with limitations of local segmental movement; and 3) Are these differences in PA movements likely to be perceivable by manual palpation?

\section{Impact that alterations in segmental mobility would be expected to have on PA movements}

Each region of the spine consists of a series of mobile segments such that a PA translational force over one vertebra will produce rotational moments between each pair of vertebrae. The moments, however, is not evenly distributed across the region but is greatest directly under the applied load.[15]

First, simple calculations were made to determine what levels of force would be most relevant for determining the size of the neutral zone. It has been suggested that the size of the neutral [16] or lax [17] zone where movement is "produced with a minimal internal resistance" [16] is the most clinically relevant aspect of joint movement. $[18,19]$ The PA force needed to reach the limits of the neutral zones were calculated from the length of the cervical and lumbar [15] spines and measurements of segmental stiffness $[15,20]$ and found to be $>10 \mathrm{~N}$ for the cervical and $>15 \mathrm{~N}$ for the lumbar spines. To place those values in perspective, clicking a retractable ball point pen typically requires $4 \mathrm{~N}-6 \mathrm{~N}$ while a plunger for hand sanitiser requires about $25 \mathrm{~N}$.

Computer-based model of the cervical spine

A simplified computer-based model was constructed to determine how increased stiffness across the entire movement of an individual segment would impact on PA movements. The model was 
constructed in SimMechanics ${ }^{\mathrm{TM}}{ }^{1}$ from published dimensional [21] and stiffness data. Movement was modelled around a single flexion/extension axis for each motion segment as constrained by linear springs as in previous models [22] and again as having non-linear angle/moment curves derived from published cadaveric data [23]. Vertebrae were modelled as massless, a minimal stiffness was applied to the neutral zone to ensure a determinant simulation, and damping was set to the minimum that would allow a smooth simulated movement. PA force was applied over the centre of rotation of flexion/extension of $\mathrm{C} 4 / 5$ in $0.1 \mathrm{~N}$ increments up to $25 \mathrm{~N} 1$ ) with normal stiffness, 2 ) with the stiffness increased throughout by $50 \%$ at $\mathrm{C} 4 / 5$, and 3 ) with the angular movement at $\mathrm{C} 3 / 4$ similarly reduced.

When the segmental kinematics were modelled as linear, the F-D curves of the PA movements were also linear. Stiffening either segment resulted in a similar increase in the slope of the linear F-D curve. When kinematics were modelled more realistically as non-linear springs, stiffening either segment resulted in the F-D curves being shifted to the left. When the stiffened segment was directly under the applied force, the curve shifted further to the left. Thus, when a segment was stiffened, the F-D curves diverged from normal primarily at forces lower than about $10 \mathrm{~N}$ and the difference was greater when the PA force was applied over the stiffened segment (Figure 1). The important differences may be more visible in the stiffness curves (see definitions) which represent the same data. The points where stiffness changes quickly are visible as 'steps' in the curves. The steps occur earlier and are higher when C4/5 was modelled as hypomobile. When the adjacent level (C3/4) was modelled as hypomobile, the effect was qualitatively similar, but much less pronounced.

\footnotetext{
${ }^{1}$ SimMechanics version 2.7, The MathWorks, Inc., Natick, Massachusetts, USA
} 


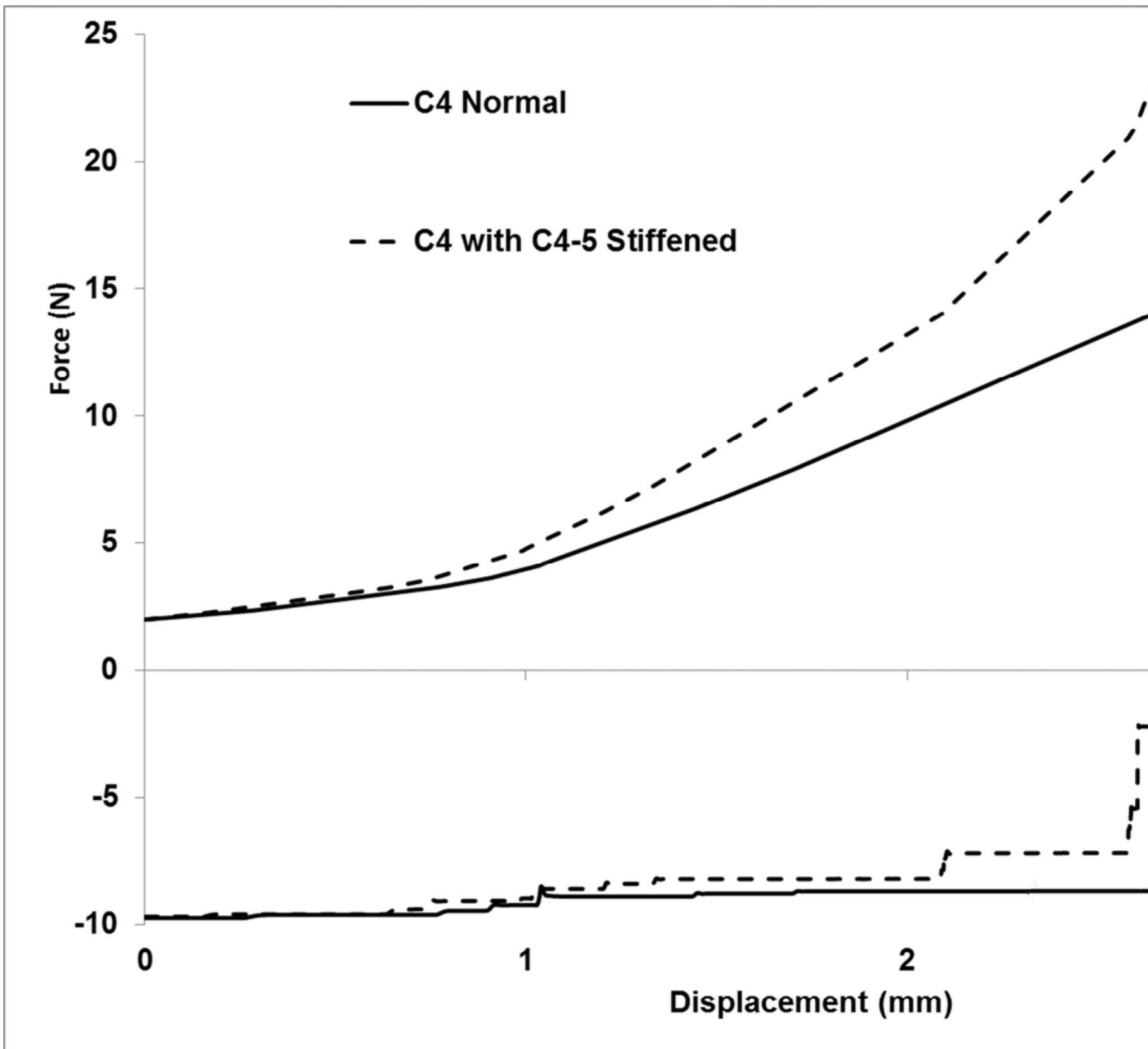

Figure 1. Graphs of the F-D and stiffness-displacement curves from the simulation with the PA force applied to $\mathrm{C} 4 / 5$ with normal movement and with C4/5 stiffened by $50 \%$.

Physical model of the lumbar spine

A physical model was constructed and used to clarify how altered segmental movements would impact on PA movements. A custom lumbar spine model was developed based on a commercially available biomechanical lumbar spine model ${ }^{2}$. The original model was developed to test surgical implants and is accurate at high loads, but does not effectively simulate movement at low loads. The custom model was comparable with published data for both the low and high loads and was modified to enable stiffening of individual motion segments (Figure 2).

\footnotetext{
${ }^{2}$ Sawbones ${ }^{\circledR}$,Vashon Island, Washington, USA
} 


\section{ACCEPTED MANUSCRIPT}

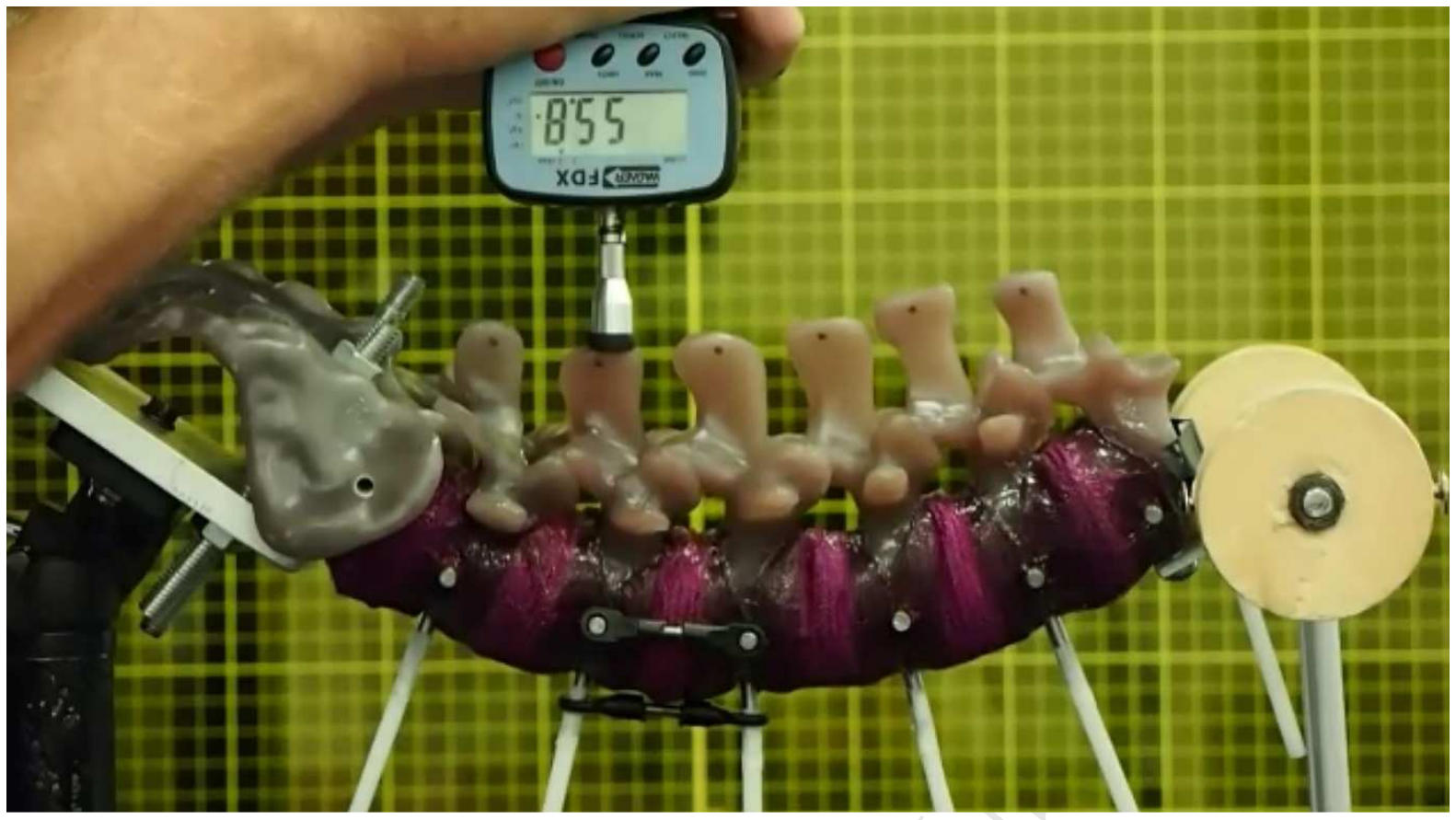

Figure 2. The experimental method with the biomechanical lumbar spine model.

The model was suspended from the sacrum and T12. Video recordings from 2.4M away included a calibration scale at the beginning of each clip. Three repetitions of PA force were applied to L 4 with a $1 \mathrm{~cm}^{2}$ rubber tipped applicator on a Wagner FDX $X^{\mathrm{TM}}$ force gauge in three conditions: first with the model unconstrained; second with L3/4 stiffened; and finally with $\mathrm{L} 2 / 3$ stiffened. Force data was read from each image where the reading on the force gauge updated. Displacements on the corresponding frames were calculated by tracking a dot on the L4 spinous process of using MtrackJ [24] , a manual tracking plugin for ImageJ. Each F-D curve was interpolated using one way splines to $0.1 \mathrm{~N}$ intervals ${ }^{3}$. The average displacement for each level of force was then calculated and this curve used in further analysis. Stiffness was calculated as the slope of the averaged curve (Figure 3).

\footnotetext{
${ }^{3}$ (SRS1 Cubic Spline for Excel ${ }^{\circledR}$ (version 2.51) http://www.srs1software.com/SRS1CubicSplineForExcel.aspx)
} 


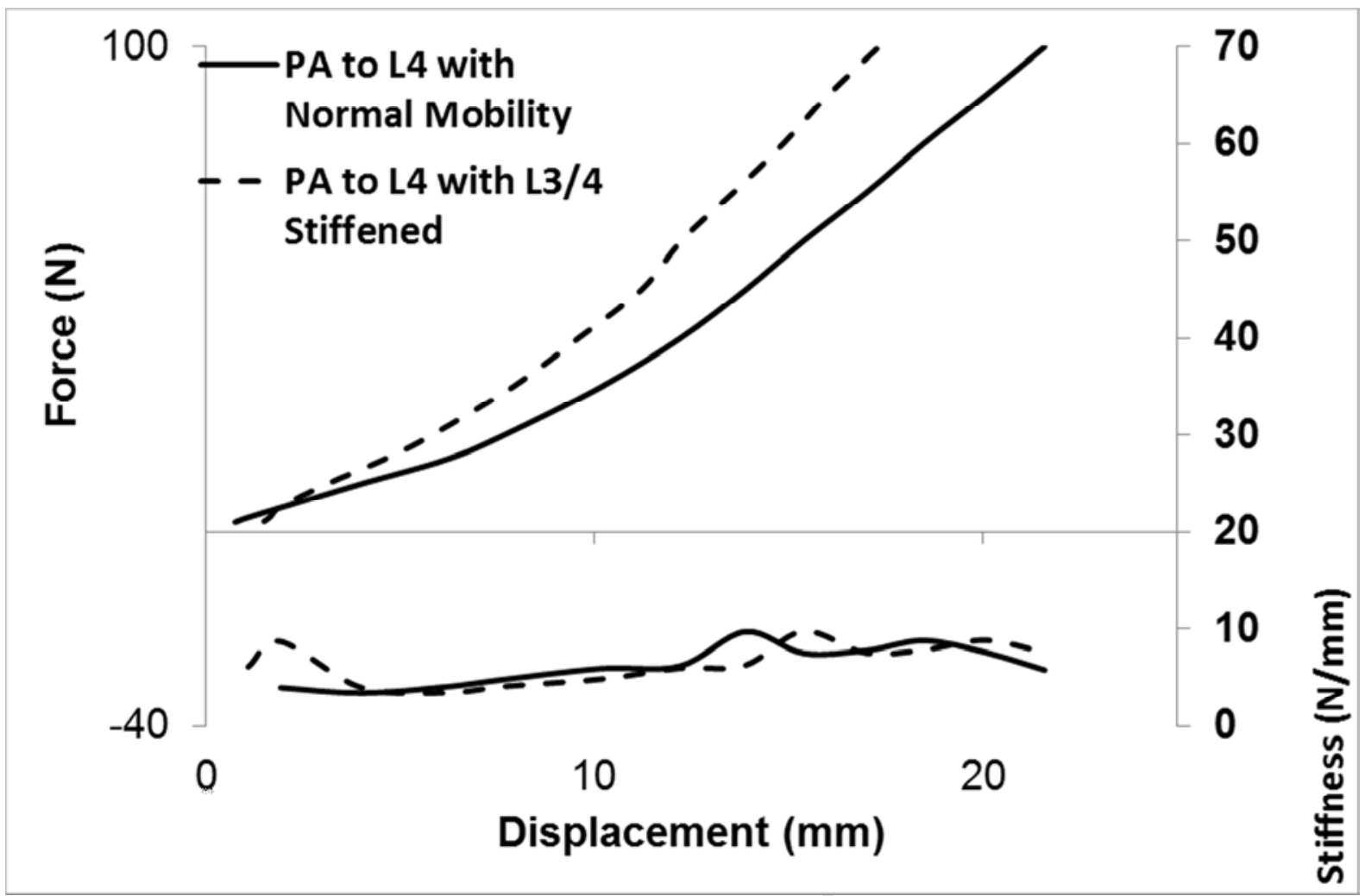

Figure 3. The resultant F-D and stiffness-displacement curves from the lumbar spine simulation.

Similar to the computer-based modelling, when a motion segment was stiffened, the F-D curve shifted to the left such that the curves diverged starting at a few newtons force and approached parallel at higher forces. Again, the effects were much more pronounced when the stiffened segment was under the applied force. The stiffness curves show stiffness increased by over $100 \%$ at displacements of a few $\mathrm{mm}$ and forces less than $10 \mathrm{~N}$.

Implications

The computational and physical models explored above demonstrate consistent findings on how PA movements would be expected to change if an underlying motion segment was hypomobile. Movement of the entire lax zone of the motion segment underlying the applied PA would be completed by PA forces of 10-15N. Similarly, the computer-based modelling indicated that the curves of PA movements and the location of points where the change in stiffness is more sudden would be most affected at forces below 10-15N. The first and largest of these points occurred at forces under five $\mathrm{N}$ for the cervical spine and under $10 \mathrm{~N}$ for the lumbar spine, each with differences in stiffness between the normal and stiffened conditions of over $100 \%$.

\section{Comparison of PA movements in clinical situations with expectations} from modelling

PA movements of the thoracic spine

PA movements were compared for two locations of thoracic spines that were perceived as having different degrees of mobility. Ten healthy, asymptomatic female participants, aged 18-25 years volunteered for a one hour session in a university setting between May and June 2017. Each was 
assessed by an experienced physiotherapist and two spinous processes between T3 and T7 were selected and marked on the skin; one considered 'hypomobile' and the other to have normal mobility. A method similar to that used for the lumbar spine model above was used to measure PA movements (Figure 4) The main differences in methodologies were that the maximum force was now $40 \mathrm{~N}$ and Kinovea $^{\mathrm{TM}^{4}}$ software was used as it allowed easier automated tracking. Investigators who applied the PA movement and did the processing were blinded to how locations were classified. Ethics approval for this study was obtained from $x x$.

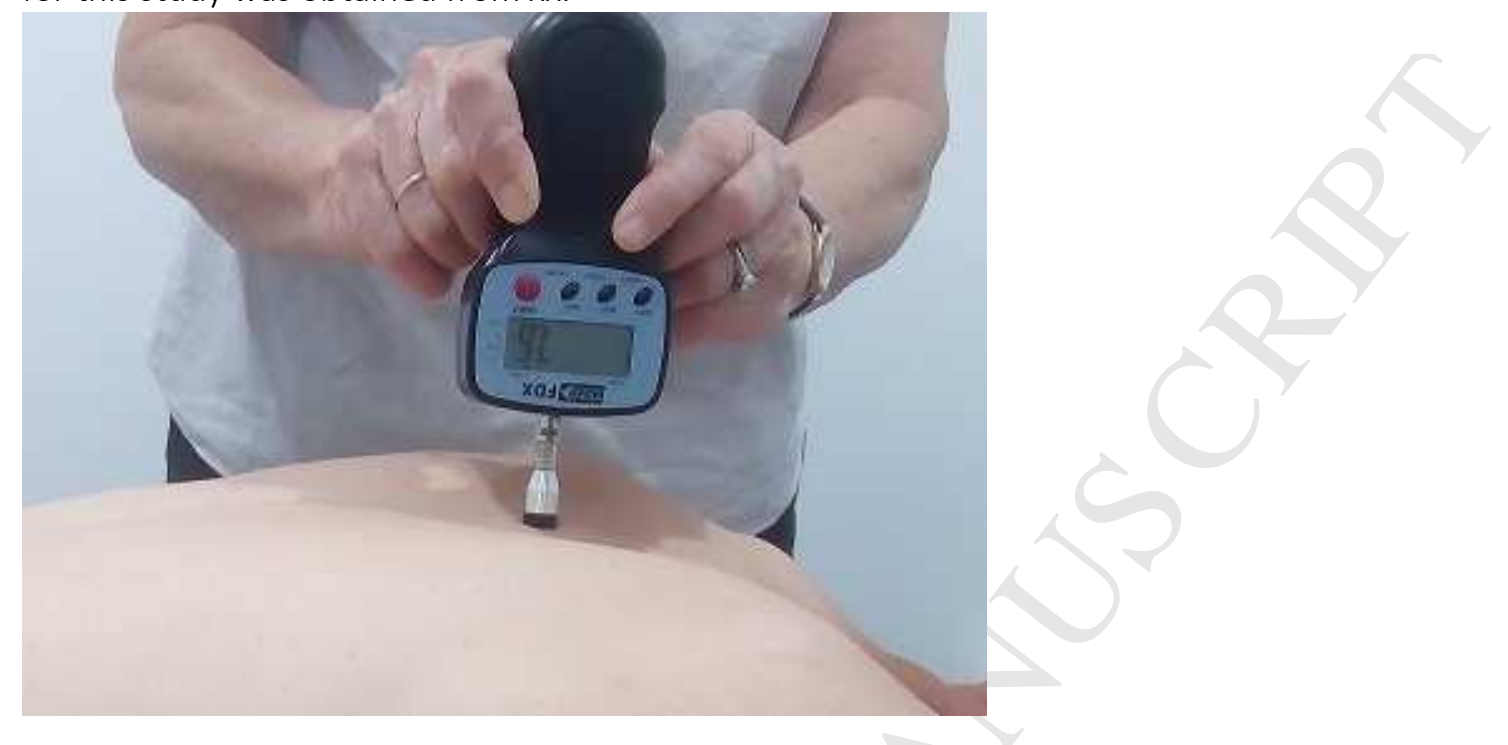

Figure 4. Method of measuring PA movements to the thoracic spine in vivo. The PA force was applied perpendicular to the surface of the skin and displacement measured by the Kinovea ${ }^{\mathrm{TM}}$ software from the video.

The hypomobile locations were not related to actual or relative spinal level. For nine out of the ten participants, the curve for the location considered to be hypomobile was shifted to the left by a significant distance (greater than the limits of agreement of the comparison level). The greatest divergence was in the regions below $15 \mathrm{~N}$ (Figure 5). The stiffness curves show the hypomobile locations generally had a peak stiffness that occurred earlier in the range of movement and was larger.

\footnotetext{
${ }^{4}$ https://www.kinovea.org/
} 


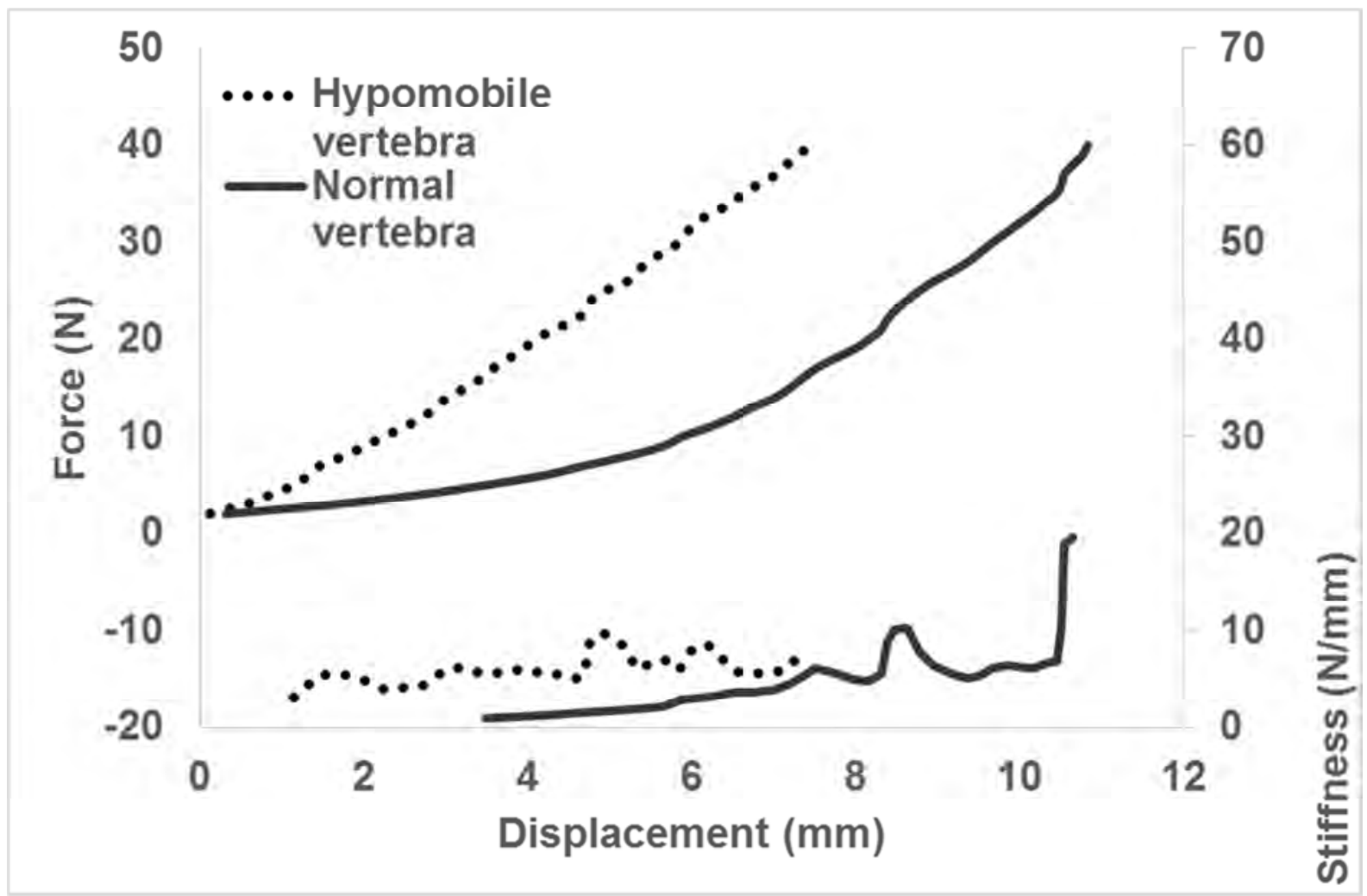

Figure 5. Representative data as captured from the method in Figure 4. F-D curves for PAs to one spinous process deemed to be hypomobile and another to be more mobile are demonstrated.

PA movements of the cervical spine

Two previous studies considered how PA movements of the cervical spine change with local tenderness [25] and when patient range of motion improves. [26] The method and high repeatability of measurement of F-D curves used in these studies is described elsewhere.[27] Briefly, a load cell and linear displacement transducer were used to measure force and displacement of manually applied unilateral PA movements.

In the first study [25], tenderness was evaluated in much the same way as would occur clinically, but in an asymptomatic population. F-D curves of PA movements to the two sides of the same vertebra were compared when the tenderness differed between sides. The soft tissue was displaced prior to applying a PA force over the articular pillar. The differences between the sides were similar to the thoracic spine with most curves for the more tender sides shifted to the left. On average, the more tender sides were also significantly stiffer between 12 and $16 \mathrm{~N}$.

In the second study[26], changes in active range of movement and passive PA movements were measured before and after treatment in a symptomatic population. Following two minutes of localised manual therapy treatment, PA stiffness decreased by up to $8 \%$ at forces of $4-7 \mathrm{~N}$ and $14-17 \mathrm{~N}$ only at the treated location. Decreases in PA stiffness also correlated with increases in active ROM and all participants who had an increase in ROM also had a decrease in PA stiffness. These changes in PA stiffness following treatment are similar to the changes in stiffness of passive rotation following treatment in a separate study.[28]

Summary of in vivo studies 
For the studies in the thoracic and cervical spines, the differences in F-D curves are compatible with what would have been expected from the modelling described above. The presence of soft tissues or differences in technique may have accounted for the higher force levels where differences in stiffness occurred in the cervical spine than would have been predicted from the modelling. Also, unilateral PA pressures were applied to the side of one vertebrae rather than centrally as in the simplified models.

\section{Are differences in PA movements likely to be perceivable by manual palpation?}

This section considers whether differences in PA movements predicted from the modelling and found in the studies described above would be perceivable by manual palpation. Firstly, for differences to be perceptible the stimuli must be above sensory thresholds. Measurements of sensory thresholds of the fingers have ranged from 0.03 to $0.05 \mathrm{~N}$ [29] which is two orders of magnitude below the forces in PA movements where the relevant differences in stiffness occur. Secondly, therapists must have sufficient motor control to produce the necessary movements. Trained persons can perform such procedures with high reliability and minimal error of applied forces as low as 2-5 N [30, 31], approximately half of the force at which the maximum changes in stiffness would be expected to occur.

Finally, the differences must be detectable. In order to be detectable, the differences must be greater than the difference threshold or just noticeable difference (JND). The size of a JND is generally proportionate to the magnitude of the stimulus. This relationship is referred to as a Weber Fraction; as named for Ernst Weber, who investigated the JND of multiple sensory functions. In other words, a given magnitude of difference in stiffness is easier to detect at low levels of stiffness. For non-linear stiffness as occurs in PA movements, the low levels of stiffness occur at low levels of force.

The Weber fractions for the ability of therapists to discriminate differences in stiffness in the range that occurs in PA movements have been found to be between 4\% [32] and 11\% [33]. These studies were, however different from what actually occurs in PA movements in two important ways. First, the movements being differentiated had simple, linear F-D curves rather than the complex curves that occur in PA movements. Secondly, the force was applied to rigid or slightly padded surfaces limiting the information the therapists could gain from soft tissue deformation of their hands. Previous studies suggest much greater sensitivity when cutaneous information is available by the fingers directly contacting deformable surfaces [34]. It is difficult to confidently quantify ability to discriminate stiffness in the clinical setting, but considering the methodologies described above, their estimates of JNDs are likely to be conservative.

The differences in stiffness the differences in stiffness predicted from the modelling and found in the in vivo studies are all significantly greater than $10 \%$ so should be easily perceivable by manual assessment. Importantly, it is also likely that therapists could discriminate several levels of stiffness between the extremes described in the previous sections.

One study has evaluated therapists' ability to perceive differences that are directly related to those that occur with PA movements. [35] A mechanical model of the cervical spine was developed to determine the ability of examiners to perceive subtle differences in stiffness and motion with PA movements as might occur in vivo. The model was different from previous devices in that it incorporated three-point bending, allowing multi-segmental movement when pressure was applied to a single simulated vertebra and had both viscoelastic characteristics and a neutral zone of each segment that could be adjusted. The model was specifically designed to replicate the F-D curves of the human cervical spine [36]. The difference in force applied to shift the F-D curve by 2 to $4 \mathrm{~mm}$ was 
less than $5 \mathrm{~N}$. Physiotherapy students and clinicians detected changes in mobility and stiffness demonstrated by shifts in F-D curves of PA-type movements of 2 and $4 \mathrm{~mm}$. Individual performances of accuracy varied widely with one experienced practitioner being $96 \%$ accurate. During data collection, the subjects used a wide variety of methods and strategic approaches to accomplish the task and, much like in clinical practice, this may contribute to the variability in sensitivity.

In summary, there is sufficient evidence to suggest humans, particularly trained at the task, are capable of detecting differences smaller than would be expected in PA movements when the underlying segment has limited mobility. The relatively low inter-rater reliability values with PIVMs, including PAs, reported in numerous studies may well have explanations other than the innate capacities of humans, particularly the capacities of skilled manual therapists.

\section{Discussion}

The modelling studies suggest the impact a reduction in segmental mobility would be expected to have on PA movements. The PA curves from the three in vivo studies demonstrated similar differences to those that would be expected from the modelling studies. The expected and found differences were of a magnitude that should be detectable by manual palpation.

For both the modelling and in vivo studies, the F-D curves for PA movements were shifted to the left such that they primarily diverge from the normal curve early in the movement. In other words, the point where the slope of the F-D curve first increased occurred earlier in the movement. When the same data are viewed as stiffness-displacement curves, the points of maximum increase in stiffness occurred earlier in the curves at forces below $10-20 \mathrm{~N}$.

The utility of passive intervertebral movements in clinical decision making has received noteworthy criticism [37-41], largely based on reported low inter-rater reliability studies, impact on patient outcomes, and the potential for bias. Several issues are present, however, with these studies and the presumption to be dismissive of the value of the performance and interpretation of PIVMs fails to consider all the available evidence. Several of these studies employed practitioners educated and trained with differing philosophies and methodologies. Multiple PIVM techniques have been homogenously analysed within individual studies and reviews [42, 43]. Other studies used subjects for palpation who were asymptomatic and, thus, perhaps lacking the movement aberrations sought for analysis by the examiners [44, 45]. Failure to place PIVM in a clinical context is also problematic. For example, reliability is higher when therapists make clinically relevant decisions such as comparisons rather than classifications [37] and studies whose context was more similar to clinical practice demonstrated better reliability. Practitioners who had similar training and performed similar techniques also demonstrated higher inter-rater reliability values [37,46]. Additionally, the design of several studies failed to consider that the repeated application of passive movement to the spines of the individuals receiving PIVMs could change the test conditions across subjects conducting the examinations; the testing, in effect, becoming treatment and reducing or eliminating the basis for comparison across subjects [42, 44, 47-51]. Finally, it is probably reasonable to not expect a higher level of agreement from PIVM movements than other assessment procedures as, for example, few cervical spine examination procedures in isolation demonstrate high repeatability [52].

As distinct from reliability, perhaps the clearest summary of the clinical relevance of the relationship between PIVM testing and clinical outcomes is found in a recent review relating to the lumbar spine [53], which concluded amongst other things that 1) Patients with low back pain may have increased PA stiffness, 2) PA stiffness decreases as symptoms improve, at least in some patients, 3) Patients 
with increased PA stiffness are probably more likely to benefit from manipulative treatment, 4) there are a wide variety of factors that influence PA stiffness.

While beyond the scope of this paper to discuss all of the evidence, it is worthwhile considering a few important differences in the approach of the current paper. Previous modelling of the relationship between PA and segmental movements of the spine represented movement characteristics as linear springs.[54] The current modelling demonstrated that such simplistic modelling produces qualitatively different responses than a more accurate non-linear representation. Specifically, with linear modelling, segmental stiffness changes in PA movements are not localisable to the underlying segment; whereas with non-linear segmental stiffness, PA movements are preferentially affected when applied over a stiffened segment.

Most previous studies that measured and compared PA movements in clinically relevant settings, focused on the stiffness near the end of range. A review of previous studies[53] indicated that a variety of methods and rationale were used to reduce the data in the curves to one or two values that were thought to represent the overall mobility. Although it has been clearly demonstrated that no region of the F-D curves of PA movements are linear[55], these studies calculated stiffness as a linear approximation of a portion of the F-D curves. Most of the studies considered a single measure of the 'linear' region of the curve late in range, ignoring the early portion of the curves that the authors suggest is most relevant. A few clinical studies [56] also considered movement in the early part of the PA movement. Although described as the displacement up to a specified level of force - typically $30 \mathrm{~N}$ for the lumbar spine - this measurement is also simply an indication of the average stiffness from the lowest force where data was collected to the cut-off force. Most of the studies described in this paper considered the behaviour of the entire curves to be able to understand where differences actually occurred rather than limiting the analysis to aspects of curves that were thought $a$ priori to be relevant.

A significant consideration of clinical context is that all of the aforementioned studies consider the performance of PIVMs, and simulations thereof, as only a manual execution and perception-based task. Under such pretense, these procedures are devoid of any potentially augmentative inputs ranging from subtle nonverbal feedback from the patient to overt expressions of pain as often occur with PIVMs. In common clinical practice, the sensorimotor experience by the practitioner in completing PIVMs is integrated with practitioner-patient interaction of many dimensions in the clinical reasoning processes inherent in patient care. The perspective offered in this paper necessarily focuses on the performance and perception aspect in isolation and, thus, must be understood within that limited scope. The execution and interpretation of PIVMs, including PAs, within patientpractitioner interaction, however, is multi-faceted and extends beyond attempts to manually assess tissue stiffness.

\section{Limitations}

It is important to emphasise that the findings discussed above do not indicate that alterations in segmental stiffness necessarily exist or are relevant to patient symptoms, but simply that PA movements can behave 'as if' there are local limitations of mobility. Neither do these findings suggest a mechanism for the production of segmental stiffness if it does exist nor do the findings have implications for the level of force used in treatment. The authors also do not suggest that the presence of the characteristics of PA movements described above necessarily indicate that location to be symptomatic, nor that PA movements to all symptomatic locations demonstrate the characteristics described above.

\section{Summary and conclusions}


Differences in PA movements that occur with tender locations in the cervical spine, perceived stiffness in the thoracic spine and improvement in patient symptoms in the cervical spine are all consistent with what would be expected in the presence of reduced segmental mobility. These differences, as suggested by the F-D curves, are most apparent and likely most detectable at levels of force (10-20N) below that often emphasized in teaching manual assessment. Thus, teaching and learning manual examination skills may benefit from highlighting the importance on stiffness and movement ease with less force application, consistent with detection of R1.

To enhance discriminatory ability, a three-step methodology of the manual examination is recommended. A 'first pass' screening is performed to the entire region at very low forces (typically without any patient discomfort) to determine a small number of locations that may be of interest. Secondly, these areas of interest are explored with slightly more force and the patient's subjective responses considered, determining which locations are related to the patient's symptoms. Finally, a location considered to be a relevant target for treatment is explored more fully considering both pain and stiffness responses to inform treatment decisions. This tiered approach of integration of manual examination and assessment may simplify learning discriminatory tasks and related clinical decisions.

A future article will consider the clinical implications of the findings presented in this article and further research is underway to clarify the relationships of PA movements to clinical practice.

Acknowledgements: We would like to thank Mitchel Jacobs, Chris Kiley, Sean Spillane, and Grace Wheeler for their contribution to testing the lumbar spine model; and Koby Byrne, Nathan Frankland, Benjamin Gehrke, and Ethan Hanley for their work on thoracic spine measurements. No funding was received for any of the studies described.

\section{Definitions:}

Force displacement curves are the force plotted against the translational displacement produced by that force. Movement diagrams are typically intended to be force displacement curves. The accuracy of movement diagrams is questionable since the F-D curves drawn by therapists of known materials bear little resemblance to measured curves.[1]

Stiffness is the change in force per unit change in displacement. In other words stiffness is the slope or first derivative of force displacement curves. Compliance, which is the inverse of stiffness is sometimes used to indicate a comparable perception.

Resistance in PA movements is the reaction force exerted by the tissue in response to the movement and is therefore equal to the PA force.

$\mathrm{R} 1$ is defined as the first perception of resistance. It has been accurately suggested that since some force is required to produce and PA movement, the idea of a first onset of resistance according to this definition is meaningless [57]. We would suggest that what is typically referred to as R1 corresponds to a change in the slope of the force displacement curve similar to what has been used to define the limit of the lax or neutral zone in passive physiological movements $[17,58]$.

Keywords: Passive movements; spinal assessment; psychomotor skills;

1. Grieve, G.P., Common vertebral joint problems. 1981, Melbourne: Churchill Livingstone.

2. Kulig, K., R. Landel, and C.M. Powers, Assessment of lumbar spine kinematics using dynamic MRI: a proposed mechanism of sagittal plane motion induced by manual posterior-to- 
anterior mobilization. Journal of Orthopaedic \& Sports Physical Therapy, 2004. 34(2): p. 5764.

3. Lee, R. and J. Evans, An in vivo study of the intervertebral movements produced by posteroanterior mobilization. Clinical Biomechanics, 1997. 12(6): p. 400-408.

4. Lee, R.Y., et al., Dynamic response of the cervical spine to posteroanterior mobilisation. Clinical Biomechanics, 2005. 20(2): p. 228-31.

5. Caling, B. and M. Lee, Effect of direction of applied mobilization force on the posteroanterior response in the lumbar spine. Journal of Manipulative and Physiological Therapeutics, 2001. 24(2): p. 71-8.

6. McGregor, A.H., et al., Cervical spine mobilizations in subjects with chronic neck problems: An interventional MRI study. Journal of Back and Musculoskeletal Rehabilitation, 2005. 18(1-2): p. 21-28.

7. Powers, C.M., et al., Segmental mobility of the lumbar spine during a posterior to anterior mobilization: assessment using dynamic MRI. Clinical Biomechanics, 2003. 18(1): p. 80-3.

8. Gal, J., et al., Measurements of vertebral translations using bone pins, surface markers and accelerometers. Clinical Biomechanics, 1997. 12(5): p. 337-340.

9. Gal, J., et al., Movements of vertebrae during manipulative thrusts to unembalmed human cadavers. Journal of Manipulative and Physiological Therapeutics, 1997. 20(1): p. 30-40.

10. Sran, M.M., et al., Posteroanterior stiffness predicts sagittal plane midthoracic range of motion and three-dimensional flexibility in cadaveric spine segments. Clinical Biomechanics, 2005. 20(8): p. 806-12.

11. Lee, M., et al., Towards a theory of lumbar mobilisation-the relationship between applied manual force and movements of the spine. Manual Therapy, 2000. 1(2): p. 67-75.

12. Chansirinukor, W., M. Lee, and J. Latimer, Contribution of ribcage movement to thoracolumbar posteroanterior stiffness. Journal of Manipulative and Physiological Therapeutics, 2003. 26(3): p. 176-83.

13. Kawchuk, G.N. and O.R. Fauvel, Sources of variation in spinal indentation testing: indentation site relocation, intraabdominal pressure, subject movement, muscular response, and stiffness estimation. Journal of Manipulative and Physiological Therapeutics, 2001. 24(2): p. 84-91.

14. Lee, M., J. Gal, and W. Herzog, Biomechanics of manual therapy, in Clinical Biomechanics, Z. Dvir, Editor. 2000, Churchill Livingstone: Philadelphia. p. 209-237.

15. Lee, R.Y.W., et al., Bending stiffness of the lumbar spine subjected to posteroanterior manipulative force. Journal of Rehabilitation Research and Development, 2005. 42(2): $p$. 167-174.

16. Panjabi, M.M., The stabilizing system of the spine. Part II. Neutral zone and instability hypothesis. Journal of spinal disorders, 1992. 5: p. 390-390.

17. Crawford, N.R., J.D. Peles, and C.A. Dickman, The spinal lax zone and neutral zone: measurement techniques and parameter comparisons. Journal of Spinal Disorders, 1998. 11(5): p. 416-29.

18. Klein, G.N., et al., Trapped in the neutral zone: another symptom of whiplash-associated disorder? European Spine Journal, 2001. 10(2): p. 141-8.

19. Oxland, T.R. and M.M. Panjabi, The onset and progression of spinal injury: a demonstration of neutral zone sensitivity. Journal of biomechanics, 1992. 25(10): p. 1165-1172.

20. Panjabi, M.M., et al., Three-dimensional load-displacement curves due to forces on the cervical spine. Journal of Orthopaedic Research, 1986. 4(2): p. 152-61.

21. Harrison, D.D., et al., Modeling of the sagittal cervical spine as a method to discriminate hypolordosis: results of elliptical and circular modeling in 72 asymptomatic subjects, 52 acute neck pain subjects, and 70 chronic neck pain subjects. Spine, 2004. 29(22): p. 2485-92.

22. Lee, M., D.W. Kelly, and G.P. Steven, A model of spine, ribcage and pelvic responses to a specific lumbar manipulative force in relaxed subjects. Journal of Biomechanics, 1995. 28(11): p. 1403-8. 
23. Panjabi, M.M., et al., Mechanical properties of the human cervical spine as shown by threedimensional load-displacement curves. Spine, 2001. 26(24): p. 2692-700.

24. Meijering, E., O. Dzyubachyk, and I. Smal, 9 Methods for Cell and Particle Tracking. Methods in enzymology, 2012. 504(9): p. 183-200.

25. Tuttle, N., R. Barrett, and L. Laakso, Posteroanterior movements in tender and less tender locations of the cervical spine. Manual Therapy, 2009. 14(1): p. 28-35.

26. Tuttle, N., R. Barrett, and L. Laakso, Relation between changes in posteroanterior stiffness and active range of movement of the cervical spine following manual therapy treatment. Spine, 2008. 33(19): p. E673-9.

27. Tuttle, N., R. Barrett, and L. Laakso, Postero-anterior movements of the cervical spine: Repeatability of force displacement curves. Manual Therapy, 2008. 13(4): p. 341-348.

28. Dugailly, P.M., et al., Analysis of the upper cervical spine stiffness during axial rotation: A comparative study among patients with tension-type headache or migraine and asymptomatic subjects. Clinical Biomechanics, 2016. 42: p. 128-133.

29. Kratchman, L.B., et al., Force Perception Thresholds in Cochlear Implantation Surgery. Audiology \& neuro-otology, 2016. 21(4): p. 244-249.

30. Oku, T. and S. Furuya, Skilful force control in expert pianists. Exp Brain Res, 2017. 235(5): p. 1603-1615.

31. Hosoda, M. and S. Furuya, Shared somatosensory and motor functions in musicians. Sci Rep, 2016. 6: p. 37632.

32. Gurari, N., A.M. Okamura, and K.J. Kuchenbecker, Perception of force and stiffness in the presence of low-frequency haptic noise. PloS one, 2017. 12(6): p. e0178605.

33. Maher, C. and R. Adams, A psychophysical evaluation of manual stiffness discrimination. Australian Journal of Physiotherapy, 1995. 41: p. 161-7.

34. Srinivasan, M.A. and R.H. LaMotte, Tactual discrimination of softness. J Neurophysiol, 1995. 73(1): p. 88-101.

35. Hazle, C.R. and A.J. Nitz, A simulated passive intervertebral motion task: observations of performance in a cross-sectional study. Journal of Manual \& Manipulative Therapy, 2012. 20(3): p. 121-129.

36. Tuttle, N., R. Barrett, and L. Laakso, Postero-anterior movements of the cervical spine: repeatability of force displacement curves. Man Ther, 2008. 13(4): p. 341-8.

37. Cooperstein, R. and M. Young, The reliability of spinal motion palpation determination of the location of the stiffest spinal site is influenced by confidence ratings: a secondary analysis of three studies. Chiropr Man Therap, 2016. 24: p. 50.

38. Haas, M., et al., Efficacy of cervical endplay assessment as an indicator for spinal manipulation. Spine (Phila Pa 1976), 2003. 28(11): p. 1091-6; discussion 1096.

39. Huijbregts, P., Clinical Prediction Rules: Time to Sacrifice the Holy Cow of Specificity? The Journal of Manual \& Manipulative Therapy, 2007. 15(1): p. 5-8.

40. Troyanovich, S.J., D.D. Harrison, and D. Harrison, Motion palpation: it's time to accept the evidence. Journal of manipulative and physiological therapeutics, 1998. 21(8): p. 568.

41. King, W., et al., The validity of manual examination in assessing patients with neck pain. Spine J, 2007. 7(1): p. 22-6.

42. Piva, S.R., et al., Inter-tester reliability of passive intervertebral and active movements of the cervical spine. Man Ther, 2006. 11(4): p. 321-30.

43. Seffinger, M.A., et al., Reliability of spinal palpation for diagnosis of back and neck pain: $a$ systematic review of the literature. Spine (Phila Pa 1976), 2004. 29(19): p. E413-25.

44. Strender, L.E., M. Lundin, and K. Nell, Interexaminer reliability in physical examination of the neck. J Manipulative Physiol Ther, 1997. 20(8): p. 516-20.

45. Fjellner, A., et al., Interexaminer reliability in physical examination of the cervical spine. J Manipulative Physiol Ther, 1999. 22(8): p. 511-6. 
46. Marcotte, J., M.C. Normand, and P. Black, The kinematics of motion palpation and its effect on the reliability for cervical spine rotation. J Manipulative Physiol Ther, 2002. 25(7): p. E7.

47. Smedmark, V., M. Wallin, and I. Arvidsson, Inter-examiner reliability in assessing passive intervertebral motion of the cervical spine. Man Ther, 2000. 5(2): p. 97-101.

48. Schops, P., M. Pfingsten, and U. Siebert, [Reliability of manual medical examination techniques of the cervical spine. Study of quality assurance in manual diagnosis]. Z Orthop Ihre Grenzgeb, 2000. 138(1): p. 2-7.

49. Pool, J.J., et al., The interexaminer reproducibility of physical examination of the cervical spine. J Manipulative Physiol Ther, 2004. 27(2): p. 84-90.

50. Nansel, D.D., et al., Interexaminer concordance in detecting joint-play asymmetries in the cervical spines of otherwise asymptomatic subjects. J Manipulative Physiol Ther, 1989. 12(6): p. 428-33.

51. Deboer, K.F., et al., Reliability study of detection of somatic dysfunctions in the cervical spine. J Manipulative Physiol Ther, 1985. 8(1): p. 9-16.

52. Nyberg, R.E. and A. Russell Smith, Jr., The science of spinal motion palpation: a review and update with implications for assessment and intervention. J Man Manip Ther, 2013. 21(3): p. 160-7.

53. Wong, A.Y. and G.N. Kawchuk, The clinical value of assessing lumbar posteroanterior segmental stiffness: a narrative review of manual and instrumented methods. PM\&R, 2017. 9(8): p. 816-830.

54. Lee, M., D. Kelly, and G. Steven, A model of spine, ribcage and pelvic responses to a specific lumbar manipulative force in relaxed subjects. Journal of biomechanics, 1995. 28(11): p. 1403-1408.

55. Nicholson, L., et al., Stiffness properties of the human lumbar spine: a lumped parameter model. Clinical Biomechanics, 2001. 16(4): p. 285-92.

56. Latimer, J., et al., An investigation of the relationship between low back pain and lumbar posteroanterior stiffness. Journal of Manipulative and Physiological Therapeutics, 1996. 19(9): p. 587-91.

57. Petty, N.J., et al., Manual examination of accessory movements--seeking R1. Manual Therapy, 2002. 7(1): p. 39-43.

58. Thompson, R.E., T.M. Barker, and M.J. Pearcy, Defining the Neutral Zone of sheep intervertebral joints during dynamic motions: an in vitro study. Clinical Biomechanics, 2003. 18(2): p. 89-98. 
Highlights

Spinal PA movements, while non-specific, emphasize single vertebral level motion.

Differences in PA motions of the spine are perceptible by manual examination.

Application of less force in examination may enhance discriminatory ability.

Students and novice clinicians may benefit from a tiered manual exam methodology. 


\section{Accepted Manuscript}

Spinal PA movements behave 'as if' there are limitations of local segmental mobility and are large enough to be perceivable by manual palpation: A synthesis of the literature

Neil Tuttle, Charles Hazle

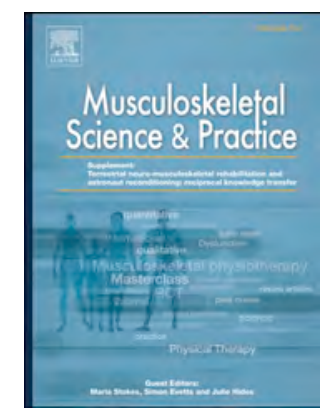

PII:

S2468-7812(18)30128-0

DOI: 10.1016/j.msksp.2018.04.005

Reference: $\quad$ MSKSP 182

To appear in: Musculoskeletal Science and Practice

Received Date: 16 February 2018

Revised Date: 10 April 2018

Accepted Date: 13 April 2018

Please cite this article as: Tuttle, N., Hazle, C., Spinal PA movements behave 'as if' there are limitations of local segmental mobility and are large enough to be perceivable by manual palpation: A synthesis of the literatureDefinitions, Musculoskeletal Science and Practice (2018), doi: 10.1016/ j.msksp.2018.04.005.

This is a PDF file of an unedited manuscript that has been accepted for publication. As a service to our customers we are providing this early version of the manuscript. The manuscript will undergo copyediting, typesetting, and review of the resulting proof before it is published in its final form. Please note that during the production process errors may be discovered which could affect the content, and all legal disclaimers that apply to the journal pertain. 